\title{
Ethics of controlled human infection to study COVID-19
}

\author{
Seema K. Shah, Franklin G. Miller, Thomas C. Darton, Devan Duenas, Claudia Emerson, Holly Fernandez \\ Lynch, Euzebiusz Jamrozik, Nancy S. Jecker, Dorcas Kamuya, Melissa Kapulu, Jonathan Kimmelman, Douglas \\ MacKay, Matthew J. Memoli, Sean C. Murphy, Ricardo Palacios, Thomas L. Richie, Meta Roestenberg, Abha \\ Saxena, Katherine Saylor, Michael J. Selgelid, Vina Vaswani, Annette Rid
}

Author affiliations are listed in the supplementary materials.

Email: seema.shah@northwestern.edu

High social value is fundamental to justifying these studies

Development of an effective vaccine is the clearest path to controlling the coronavirus disease 2019 (COVID-19) pandemic. To accelerate vaccine development, some researchers are pursuing, and thousands of people have expressed interest in participating in, controlled human infection studies (CHIs) with severe acute respiratory syndrome-coronavirus 2 (SARS-CoV-2) (1, 2). In CHIs, a small number of participants are deliberately exposed to a pathogen to study infection and gather preliminary efficacy data on experimental vaccines or treatments. We have been developing a comprehensive, stateof-the-art ethical framework for CHIs that emphasizes their social value as fundamental to justifying these studies. The ethics of CHIs in general are underexplored $(3,4)$, and ethical examinations of SARS-CoV-2 CHIs have largely focused on whether the risks are acceptable and participants could give valid informed consent (1). The high social value of such CHIs has generally been assumed. Based on our framework, we agree on the ethical conditions for conducting SARS-CoV-2 CHIs (see the table). We differ on whether the social value of such CHIs is sufficient to justify the risks at present, given uncertainty about both in a rapidly evolving situation; yet we see none of our disagreements as insurmountable. We provide ethical guidance for research sponsors, communities, participants, and the essential independent reviewers considering SARS-CoV-2 CHIs.

\section{SUFFICIENT SOCIAL VALUE}

CHIs have a long, complicated history. They have contributed to substantial improvements in clinical and public health practice, including the recent licensure of two vaccines (5), but also involved some unethical research (3). The first step in justifying SARS-CoV-2 CHIs, especially as they would involve major uncertainty and controversy, is to demonstrate their high social value. Crucially, SARS-CoV-2 CHIs should address relevant, unresolved scientific questions in rigorously designed and conducted experiments.

SARS-CoV-2 CHIs could have high social value in several ways. For example, they could help prioritize among the almost 100 investigational vaccines and over 100 experimental treatments for COVID-19 currently in development. CHIs could help identify the most promising agents, which would inform the design of larger trials, guide decisions to scale up manufacturing early, and thereby accelerate product development and implementation. If they saved even a few months of vaccine development (1), SARS-CoV-2 CHIs would contribute to faster control of the pandemic and reduce the need for, and associated costs of, physical distancing measures, providing substantial benefits for much of the world's population (including the most vulnerable).

To achieve high social value in this way, coordination of stakeholders is essential. Sponsors of SARS-CoV-2 CHIs should delineate a credible path forward from CHIs to rigorous field studies, and eventually toward scaled-up production. This is a considerable challenge given the rapidly evolving research response to the pandemic; many approaches to accelerating product development are already appropriately being pursued in parallel. It is therefore essential to plan and evaluate SARS-CoV-2 CHIs as a complement, not an alternative, to these other approaches and ensure that CHI results are integrated into the dynamic COVID-19 research landscape. For example, the World Health Organization is convening sponsors of SARS-CoV-2 CHIs to increase transparency and promote coordination. Research sponsors should lead by establishing and enforcing standards for rapid data collection, dissemination, and sharing that permit aggregation of results across CHIs. Medical journals should require compliance with these standards before accepting manuscripts. Regulatory agencies should collaborate with sponsors, researchers and policy-makers to define how CHI data will inform or modify larger trials, licensure, and manufacturing. Finally, sponsors and governments should implement mechanisms to ensure widespread, equitable access to proven products whose development was accelerated by SARS-CoV-2 CHIs. Such wide-ranging stakeholder coordination is difficult but important to demonstrate high social value. Though not achieved for proposed Zika virus CHIs during the 2015-2016 epidemic, it did occur later (6).

SARS-CoV-2 CHIs could have high social value in other 
ways, and individual CHIs could address multiple scientific questions. For example, CHIs could clarify dynamics of infection, viral pathogenesis, and risk of vaccine pathogenesis or identify correlates of protection-all of which could inform the development and implementation of vaccines. CHIs could also illuminate poorly understood parameters for modeling the pandemic and public health responses, including who is infectious and when and how infections occurred. This information is difficult to collect by observation alone, and existing animal models do not fully replicate clinical disease seen in humans. Additionally, if the pandemic wanes before larger trials are completed, SARS-CoV-2 CHIs could be critical for advancing research until the next outbreak, as with Zika virus (6). All of these paths to high social value would require similar, extensive coordination with relevant stakeholders.

SARS-CoV-2 CHIs admittedly have limited generalizability, as they would need to be conducted with low-risk populations (see below) with a non-natural mode of infection. Therefore, although some propose replacing efficacy trials with SARS-CoV-2 CHIs (1), it is more likely that CHIs accelerate vaccine or treatment development by informing larger trials, not by making such trials redundant. Yet almost all disease models or trial designs require some extrapolation or further testing. For example, field trials with frontline workers could also accelerate vaccine development, but they would not include older, retired individuals.

Thus, there are many potential ways in which SARS-CoV2 CHIs could have high social value. Before their initiation, it is essential that the given social value is judged as compelling enough to justify its pursuit.

\section{REASONABLE RISK-BENEFIT PROFILE}

For SARS-CoV-2 CHIs to be ethically permissible, risks to participants, study personnel, and third parties should be minimized, reasonable in relation to the social value of the research, and below the upper limits of acceptable risk $(7,8)$. There are both scientific unknowns about SARS-CoV-2 and moral disagreements about upper limits to risk. Although research inherently involves uncertainty, this situation warrants a cautious approach to evaluating SARS-CoV-2 CHIs and revisiting risk-benefit judgments as new evidence emerges.

Risk minimization should focus primarily on reducing the likelihood of serious and irreversible harms. To minimize risks to participants, SARS-CoV-2 CHIs should recruit young people without underlying medical conditions who face lower mortality risks from COVID-19 (9). Key uncertainties remain regarding other potentially serious and irreversible harms of SARS-CoV-2 infection in young people, such as cardiac or neurological injury. Accordingly, long-term follow-up of CHI participants is critical.

To minimize risks to study personnel, participants should be in inpatient isolation, with contact reduced to the extent possible and robust personal protective equipment provided. Both participants and personnel should be carefully monitored, promptly managed when symptomatic, and provided any proven targeted treatments or offered enrollment into appropriate clinical trials. To minimize risks to third parties outside the research, researchers should notify public health authorities about the studies in advance and ensure that participants who withdraw take appropriate precautions to avoid spread to others.

Participants might benefit from controlled infection and/or vaccination if they become immune to SARS-CoV-2. However, the degree and duration of naturally acquired and vaccine-derived immunity are currently unknown. Some participants would also receive placebo vaccines, and most investigational vaccines prove ineffective. The potential benefits of participation thus should be given little, if any, weight. Instead, risks to participant should be justified by the social value of SARS-CoV-2 CHIs, with higher risks requiring higher social value.

Even when research has high social value and involves competent consenting adults, there is substantial consensus that risks to participants should not exceed an absolute upper limit. Regulations and ethics guidance do not clearly delineate this limit. Some commentators have argued that it should not exceed a $1 \%$ risk of death or the risks posed by activities that, like research, expose some people to risk to benefit others, such as living organ donation $(8,10)$. Although these are imperfect analogies to research, they provide helpful context for evaluating limits of acceptable research risk.

Current data on SARS-CoV-2 infection come from relatively small samples with missing data points and are still being scrutinized. Data suggest that 20- to 44-year-olds with diagnosed infection-including those with underlying conditions-have a mortality risk less than $0.2 \%$ (11). But diagnostic testing has been limited, making the number of undiagnosed infections unknown. One attempt to account for these limitations estimates that healthy adults aged 20 to 29 have a $0.03 \%$ risk of death and a 1.1\% risk of hospitalizations (9). These risks could be further reduced by refining eligibility criteria based on emerging data. Recognizing the uncertainties, risks from SARS-CoV-2 CHIs appear comparable to the risks from some other research and activities similar to research (table S1). They also seem to fall below the upper risk limits proposed for research. For third parties who could be exposed to infection from CHI participants, there is no consensus on what level of risk is acceptable (12); however, with the above safeguards, these risks could be minimized to be negligible.

\section{CONTEXT-SPECIFIC STAKEHOLDER ENGAGEMENT}

CHIs have a checkered history (3), and it can be counterintuitive for the public that researchers would infect people with 
disease-causing pathogens. Although the current pandemic context with widespread physical distancing might complicate public engagement, it remains important and feasible as SARS-CoV-2 CHIs are developed. For example, public opinion surveys could identify concerns and information deficits, and researchers could engage the media or convene virtual advisory groups. Maintaining transparency and accountability to diverse communities is important for mitigating potential mistrust, especially in a pandemic (13). As noted above, engagement with stakeholders in the research community, health professionals, and policy-makers is also critical for ensuring that the results from SARS-CoV-2 CHIs translate into social benefits.

\section{SUITABLE SITE SELECTION}

Selecting suitable sites for SARS-CoV-2 CHIs requires considering risks to participants, study personnel, and third parties; feasibility of recruitment; availability of necessary infrastructure; and potential effects on local pandemic responses. Sites should be selected for sound scientific reasons while avoiding especially vulnerable populations. For example, performing CHIs in locations with high community spread of SARS-CoV2 could be an acceptable way to reduce relative risks for participants, provided that high transmission is not due to underlying injustices. Given that participants would require testing, medical attention, and treatment, and research personnel would require personal protective equipment, sponsors should also demonstrate to ethics review boards or public health authorities that CHIs will not unduly compete for scarce resources and thereby unduly compromise the local pandemic response. All sites should have sufficient capacity to conduct rigorous studies, provide high-quality care to participants, and minimize research risks. Sites experienced with conducting CHIs might be favored to ensure that studies and local public engagement can be launched quickly, effectively, and responsibly.

\section{FAIR PARTICIPANT SELECTION}

Selecting participants fairly for SARS-CoV-2 CHIs primarily requires considering fair distribution of research risks and burdens. Because of the uncertainty and potential high risk involved, participants who are at relatively low risk of serious and irreversible harm and have capacity to give their own consent should be selected (i.e., young, healthy and competent adults).

\section{ROBUST INFORMED CONSENT}

There is widespread consensus on obtaining high-quality informed consent for CHIs and using rigorous procedures to maximize participant understanding. Evidence-based approaches to consent include requiring participants to pass a test on key study information (14). Ongoing informed consent will be important as new data emerge, notably on the risks of SARS-CoV-2 infection.

\section{PROPORTIONATE PAYMENT}

Members of our group disagree about the ethical permissibility of offering payment to CHI participants, and there may be relevant regulatory limits in different jurisdictions. Nevertheless, as SARS-CoV-2 CHIs require confinement and follow-up, fairness seems to demand offering participants compensation for their time. This may total several thousand dollars in the United States, assuming compensation at a fair minimum wage for unskilled labor, as in other CHIs. By contrast, incentives beyond compensation could be avoided, given the number of people already indicating willingness to participate. Concerns that the undue influence of monetary compensation compromises risk judgments are unsupported by the available data, as financial motivations are associated with greater attention to risk (15). Moreover, a rigorous informed consent process could maximize understanding. In case payment tempts participants to withhold disqualifying information, eligibility criteria should be objectively verifiable.

\section{CONCLUSION}

Given the extraordinary nature of the pandemic, our framework and analysis support laying the groundwork for SARSCoV-2 CHIs-for example, by developing a challenge strain, drafting consensus protocols that address ethical concerns, and engaging stakeholders to enhance their social value, minimize risks, and build public trust.

\section{REFERENCES AND NOTES}

1. N. Eyal, M. Lipsitch, P. G. Smith, Human challenge studies to accelerate coronavirus vaccine licensure. J. Infect. Dis. jiaa152 (2020). doi:10.1093/infdis/jiaa152 Medline

2. www.thecovidchallenge.org/

3. B. Bambery, M. Selgelid, C. Weijer, J. Savulescu, A. J. Pollard, Public Health Ethics 9, 92 (2016). doi:10.1093/phe/phv026 Medline

4. E. Jamrozik, M. J. Selgelid, Human Challenge Studies in Endemic Settings: Ethical and Regulatory Issues, Springer Briefs in Ethics (Springer, 2020).

5. M. Roestenberg, M.-A. Hoogerwerf, D. M. Ferreira, B. Mordmüller, M. Yazdanbakhsh, Lancet Infect. Dis. 18, e312 (2018). doj:10.1016/S14733099(18)30177-4 Medline

6. K. S. Vannice et al., Vaccine 37, 863 (2019). doi:10.1016/j.vaccine.2018.12.040 Medline

7. A. Rid, D. Wendler, Kennedy Inst. Ethics J. 21, 141 (2011). doi:10.1353/ken.2011.0007 Medline

8. F. G. Miller, S. Joffe, J. Med. Ethics 35, 445 (2009). doi:10.1136/jme.2008.026062 Medline

9. R. Verity et al., Lancet Infect. Dis. 10.1016/S1473-3099(20)30243-7 (2020). doi:10.1016/S1473-3099(20)30243-7

10. D. B. Resnik, Theor. Med. Bioeth. 33, 137 (2012). doi:10.1007/s11017-011-9201-1 Medline

11. S. Bialek et al., MMWR Morb. Mortal. Wkly. Rep. 69, 343 (2020). doi:10.15585/mmwr.mm6912e2 Medline

12. S. K. Shah et al., Science 360, 158 (2018). doi:10.1126/science.aaq0917Medline

13. AVAC, Good Participatory Practice Guidelines for HIV Prevention Research, www.avac.org/resource/good-participatory-practice-guidelines-biomedical-hivprevention-trials-second-edition. 
14. A. Nishimura et al., BMC Med. Ethics 14, 28 (2013). doi:10.1186/1472-6939-14-28 Medline

15. L. Stunkel, C. Grady, Contemp. Clin. Trials 32, 342 (2011) doi:10.1016/i.cct.2010.12.003 Medline

\section{ACKNOWLEDGMENTS}

The opinions expressed in the article are the authors' and do not reflect the views of organizations with which the authors have affiliations, including the National Institutes of Health, the Department of Health and Human Services, or the United States government. This work was primarily supported by a Making a Difference Grant from the Greenwall Foundation (S.K.S., A.R., R.P., D.D.), along with support from the Wellcome Trust (S.K.S., E.J., D.K., M.K., R.P., M.J.S., V.V.), Brocher Foundation (S.K.S., A.R., R.P., D.D., T.D.D., H.F.L., E.J., N.S.J., D.K., J.K., D.M., S.C.M., T.L.R., M.R., A.S., M.J.S., V.V.), and NIH Clinical Center Department of Bioethics (AR). The authors also thank C. Chui, K. Littler, P. Pitisuttithum, and M. Yu for their contributions, and M. Danis, C. Grady, M. Nicolini, J. Ochoa, and H. Taylor for helpful discussion.

\section{SUPPLEMENTARY MATERIALS}

www.sciencemag.org/cgi/content/full/science.abc1076/DC1

Published online 7 May 2020

10.1126/science.abc1076 


\section{Ethical framework for SARS-CoV-2 controlled human infection studies (CHIs)}

\section{SUFFICIENT SOCIAL VALUE}

Identify and address relevant, unresolved scientific questions in rigorously designed and conducted experiments

- Use rigorous methods to develop $\mathrm{CHI}$ models, including highquality manufacturing and process of challenge strains

- Define and regularly review priority scientific questions, e.g., selecting the most promising vaccine and treatment candidates; identifying correlates of protection; clarifying infection dynamics, mechanisms of disease, and possible vaccine pathogenesis

- Coordinate with stakeholders to ensure SARS-CoV-2 CHI results will affect future research, clinical, or public health practice and delineate a credible path forward for $\mathrm{CHI}$ results to make an impact

- Establish and enforce standards for data collection in SARSCoV-2 CHIs

- Share data, samples, and challenge strains appropriately

- Disseminate SARS-CoV-2 CHI results quickly through openaccess publication

Realize benefits by facilitating equitable access to proven safe and effective products

- Use mechanisms such as compulsory licensure under Trade-Related Aspects of Intellectual Property Rights (TRIPS) agreement, march-in rights against patents under U.S. Bayh-Dole Act, U.S. Food and Drug Administration's priority review voucher program

\section{REASONABLE RISK-BENEFIT PROFILE}

\section{Identify and reduce risks}

- Enroll younger, adult participants without comorbidities; refine and update eligibility criteria in light of new evidence

- Monitor closely; provide prompt, free treatment and compensation for research-related injury

- Confine participants in in-patient isolation for at least 14 days

- Coordinate with public health officials in case participants withdraw

Ensure risks do not exceed upper limits and are reasonable in relation to social value

\section{CONTEXT-SPECIFIC STAKEHOLDER ENGAGEMENT}

\section{Engage public}

- Create community advisory boards; use media to inform and engage

- Gather public input through informed opinion surveys

- Adapt engagement strategies to physical distancing as needed

Coordinate with international research, clinical, public health community

- Engage researchers, sponsors, regulators, ministries of health, etc. before, during, and after study implementation

\section{SUITABLE SITE SELECTION}

Consider feasibility of recruitment, risk, generalizability, availability of infrastructure, potential effects on local health care system

- Select location with available expertise

- Bring in extra resources so as not to unduly compromise pandemic response

\section{FAIR PARTICIPANT SELECTION}

Enroll low-risk groups with capacity to provide voluntary informed consent

- Enroll younger, adult participants without comorbidities

\section{ROBUST INFORMED CONSENT}

Ensure participant understanding

- Use evidence-based, context-specific consent materials

- Test participants on understanding of key criteria: deliberate infection; risks and burdens; potential social value; study purpose; uncertainty; restrictions on liberty to protect others

\section{PROPORTIONATE PAYMENT}

Avoid undue influence, exploitation, incentives to withhold information

- Compensate participants for their time to avoid exploitation and inequities in access to $\mathrm{CHIS}$

- Set objective, verifiable eligibility criteria (in case money tempts participants to withhold disqualifying information) 